\title{
RELACIÓN ENTRE LA MEMORIA AUDITIVA INMEDIATA Y LA COMPRENSIÓN LECTORA, EN ALUMNOS DE QUINTO Y SEXTO DE PRIMARIA DE LIMA Y HUAROCHIRÍ
}

\author{
JUAN YARINGAÑo L. ${ }^{1}$ \\ Universidad Nacional Mayor de San Marcos, Lima, Perú \\ (RECIBIDO EL 8/9/2009, ACEPTADO EL 30/11/ 2009)
}

\begin{abstract}
RESUMEN
Se estudia la relación entre la comprensión lectora y memoria auditiva inmediata en grupos de alumnos de educación primaria de Lima y Huarochirí. Los sujetos fueron seleccionados según el criterio no probabilístico intencional, conformado por 228 alumnos de instituciones educativas estatales de los distritos de San Juan de Lurigancho y San Mateo, de quinto y sexto grado de primaria y de ambos sexos, que fueron evaluados con el Test de Memoria Auditiva Inmediata y la Prueba de Complejidad Lingüística Progresiva, con el objetivo de conocer los niveles de memoria auditiva inmediata y comprensión lectora. La aplicación de los instrumentos se realizó en forma colectiva. El análisis descriptivo de los datos fue realizado mediante la distribución de frecuencias y medidas de tendencia central, y para el análisis inferencial se utilizaron las pruebas estadísticas U de Mann-Whitney y Rho de Spearman. Los resultados indicaron una correlación moderada $($ Rho $=0,430$ ) entre la comprensión lectora y la memoria auditiva inmediata. El análisis comparativo señaló diferencias estadísticas significativas en memoria auditiva inmediata por el lugar donde viven y el grado de estudio; así como en comprensión lectora según el grado de estudio. Por el contrario no se observaron diferencias significativas en memoria auditiva inmediata y en comprensión lectora según el género.
\end{abstract}

Palabras clave: Comprensión lectora, memoria auditiva inmediata, alumnos de Lima y Huarochirí.

\section{ABSTRACT}

\section{RELATIONSHIP BETWEEN STOCK AUDITORY MEMORY AND READING COMPREHENSION, IN STUDENTS OF FIFTH AND SIXTH IN LIMA PRIMARY AND HUAROCHIRÍ}

This study explores the relationship between reading comprehension and auditive memory immediate groups of students in primary education of Huarochirí and Lima. The subjects were selected according to the probabilistic approach not intentional, comprising 228 students from state schools of the district of San Juan de Lurigancho and San Mateo, fifth and sixth grade and both sexes, which were evaluated with the Test Auditive Memory Immediate and the Test of Linguistic Complexity Progressive, with the aim of knowing the levels of auditive memory immediate and reading comprehension. The implementation of the

1 Estudiante de postgrado de Psicología de la UNMSM. E-mail: juyari@yahoo.es 
instruments was conducted in a collective manner. The descriptive analysis of the data was done through the distribution of frequencies and measures of central tendency, for analysis and inferential statistical tests were used U Mann-Whitney and Spearman Rho. The results showed a correlation modest $(\mathrm{Rho}=0,430)$ between reading comprehension and auditive memory immediately. The comparative statistical analysis indicated significant differences in auditive memory instantly by where they live and degree studies, as well as in reading comprehension depending on the degree of study. By contrast not be found significant differences in auditive memory immediate and reading comprehension by gender.

Keywords: Reading comprehension, auditive memory immediately, students in Lima and Huarochirí.

\section{INTRODUCCIÓN}

Nuestro país enfrenta retos innumerables, uno de los más álgidos es el referido al ámbito educativo, donde destacan las dificultades académicas que presentan los estudiantes, en las distintas etapas de su escolaridad. Una de las áreas donde se hace más evidente estas dificultades se refiere al proceso de lectura y comprensión de textos; es así que los últimos informes y evaluaciones realizadas por instituciones nacionales e internacionales no hacen más que confirmar que los alumnos de Educación Básica no comprenden lo que leen o tienen dificultades para lograr una lectura fluida de los textos.

El Programme for International Student Assessment (Programa para la Evaluación Internacional de Estudiantes) destinado a evaluar cada tres años los conocimientos y habilidades en comprensión lectora, matemáticas y ciencias, enfatizando uno de estos tres campos en cada aplicación, la muestra estudiada la conforman alumnos de 15 años. Los resultados de las pruebas de PISA permiten situar a cada examinado en un nivel de competencia determinado, según una escala de cinco niveles en el Nivel 1, puede localizar una información puntual, captar la idea principal de un texto; Nivel 2, logra localizar información más compleja pero explícita, hacer inferencias simples; Nivel 3, integra información dispersa, captar relaciones entre partes diferentes; Nivel 4, localiza información implícita, captar matices finos, evaluar el texto y Nivel 5, dominio sofisticado de la lectura, evaluación crítica, manejo de hipótesis. A su vez se establecen tres categorías: Buenos lectores (Niveles 4 y 5), Lectores regulares (Niveles 2 y 3) y Malos lectores (Nivel 1 y menos). De acuerdo con los resultados obtenidos, el Perú ocupó el puesto 41 en las tres áreas examinadas. En el caso de la Lectura sólo el $1.1 \%$ de los alumnos peruanos alcanza niveles 4 y $5 ; 19.4 \%$ niveles 2 y 3 ; y un $79.6 \%$ presenta nivel 1 a menos. Esto resultados indican que nuestros estudiantes tienen serias dificultades para localizar información, interpretar textos y reflexionar sobre el contenido de la lectura y valorarlo (Ministerio de Educación, 2004).

A nivel nacional también se han examinado las capacidades relacionadas con la comprensión de textos y matemáticas, siendo el más reciente e importante la IV Evaluación Nacional de Rendimiento Estudiantil - 2004 (Ministerio de Educación, 2005), en la que se establecen cuatro criterios de logro: Nivel Suficiente, para los que demuestran un dominio suficiente y necesario en los desempeños evaluados para el grado que estudia; Nivel Básico, referido a estudiantes que demuestran un dominio incipiente o un manejo elemental de los desempeños desarrollados en el grado evaluado; Nivel Previo, para estudiantes que presentan un manejo de los desempeños desarrollados en grados anteriores y Nivel Anterior al Previo, en el 
caso de estudiantes que sólo pueden resolver algunas de las tareas del nivel Previo. Los resultados de esta evaluación señalan que sólo se ubican en el Nivel Suficiente el $15.1 \%$ de los alumnos de segundo grado de primaria, el $12.1 \%$ de los alumnos de sexto grado de primaria, el $15.1 \%$ de los alumnos de tercer grado de secundaria y el $9.8 \%$ de los alumnos de quinto grado de secundaria (Ministerio de Educación, 2005). En otras palabras solo 1 de cada 10 alumnos termina la Educación Básica Regular cumpliendo los objetivos educativos planteados en el área de comprensión de textos.

Estos resultados son preocupantes, dada la importancia de asegurar las capacidades de los estudiantes desde los primeros grados de la educación básica, debido a su impacto en el aprendizaje futuro. Esto pone en evidencia que los bajos desempeños no son revertidos a lo largo de la primaria y se mantienen a lo largo de la vida estudiantil. Las conclusiones de ese estudio señalan que los resultados de la Evaluación Nacional 2004, muestran problemas importantes de calidad y de equidad en los logros de los estudiantes en comprensión de textos en todos los grados evaluados, la mayoría de estudiantes no alcanza los niveles de desempeño esperados para el grado. Este problema afecta a estudiantes de todos los estratos estudiados: instituciones urbanas y rurales, estatales y no estatales, varones y mujeres. Por otro lado, en términos de equidad, los estudiantes provenientes de niveles socioeconómicos desfavorecidos también mostraron resultados bajos en los aprendizajes evaluados y los estudiantes de las instituciones educativas ubicadas en entornos rurales, obtienen resultados más bajos con respecto de aquellos que estudian en instituciones ubicadas en zonas urbanas. Asimismo al tratar de explicar estos resultados se observa que los docentes de los alumnos evaluados muestran dificultades en el dominio de algunas habilidades lectoras y que la mayoría de ellos tiene capacidad de resolver solo las tareas más sencillas. Se estableció una asociación entre los aprendizajes que muestran los estudiantes y las habilidades de sus profesores, de este modo, los alumnos de profesores con mayores habilidades en Matemática y Comunicación tienden a obtener, en promedio, mejores resultados en las pruebas respectivas (Ministerio de Educación, 2005).

Según Baddeley (1998), el estudio de la memoria a corto plazo nace con el objeto de dar respuesta a distintas cuestiones prácticas, como el intento de Jacobs a finales del siglo XIX para medir la capacidad mental de sus alumnos; el interés de Broadbent durante los años cincuenta del siglo XX en la atención dividida; o bien el interés de Conrad en los sesenta por la memorización de números de teléfono y códigos postales. Paulatinamente el estudio de la memoria a corto plazo se vincula a finales de la década de los sesenta a diferentes técnicas de laboratorio, modelos y teorías. Desde esta perspectiva, uno de los modelos multialmacén generados en los años sesenta del siglo XX, es el de Atkinson y Shiffrin, quienes reivindicaron la importancia general del almacén a corto plazo, dando por sentado que dicho almacén funcionaba como una memoria de trabajo. Aunque en trabajos posteriores Atkinson y Shiffrin apuntan datos complementarios en la línea de plantear la memoria a corto plazo como una memoria de trabajo, los modelos estructurales enfatizan el carácter temporal y unitario de este sistema de memoria, y no desarrollan en cambio los aspectos más funcionales y procesales que estarían implicados.

Si entendemos a la comprensión como una función cognitiva compleja, que permite la construcción de una representación del significado global del texto, resultado de un proceso complejo e interactivo, en el que el individuo pone en acción diferentes tipos de 
conocimientos. Nos encontramos frente a un proceso cognitivamente demandante que compromete los recursos de almacenamiento y de procesamiento de la memoria (Baddeley, 1998). De acuerdo con esta premisa se otorga un papel fundamental a la memoria a corto plazo o inmediata, en la comprensión del lenguaje oral y escrito, resaltando su presencia en los componentes fonológicos de la comprensión.

De este modo se plantea que la comprensión exige un espacio donde se vayan depositando los resultados parciales y totales del procesamiento, y donde se coordinen las restricciones simultáneas que los diferentes procesos imponen a la información compartida (García, 1987). Pero, pese a esta aceptación de la importancia de la memoria en la comprensión, aún no se han clarificado las complejas relaciones que se les atribuyen; todavía no existe acuerdo total sobre la naturaleza general o específica de este sistema y tampoco se ha dado aún una respuesta concluyente a las múltiples cuestiones que se plantean en torno a su origen y desarrollo evolutivo. Tomando esto en cuenta, se puede plantear que el proceso lector exige el uso de una memoria auditiva inmediata, que permita recordar datos, palabras o conceptos que son propuestos en el texto y que el lector verbaliza internamente (Gutiérrez, 2002).

\section{MÉTODO}

El presente es un estudio descriptivo correlacional, en la medida que describe y analiza las características de los niveles de comprensión lectora y de la memoria auditiva inmediata en un grupo de alumnos de quinto y sexto grado de primaria. También los puntajes obtenidos mediante la administración de las pruebas correspondientes son correlacionados estadísticamente (rho de Spearman), con el fin de constatar la posible relación entre los niveles de comprensión lectora y de memoria auditiva inmediata.

El grupo de sujetos participantes en la presente investigación fue seleccionado mediante el método no probabilístico intencional, teniendo en cuenta los siguientes criterios: pertenencia al ámbito se localizaron las instituciones educativas de nivel primario de gestión estatal que se encuentren ubicados en distritos pertenecientes a la provincia de Lima y de Huarochirí y estar matriculados en el 5to y 6to grado de educación primaria, sin importar su edad o sexo.

Considerando esos criterios fueron seleccionados los centros educativos: 104 alumnos del distrito de San Mateo en Huarochirí, y 124 alumnos del distrito de San Juan de Lurigancho, en Lima Metropolitana, constituyendo una muestra total de 224 estudiantes. El promedio de edad de los alumnos de quinto grado es 9,96 y de sexto grado 11,04.

El diseño de investigación, de acuerdo con los objetivos del presente estudio el diseño utilizado fue el de una investigación descriptiva comparativa. Siendo las variables de estudio.

- Nivel de memoria auditiva inmediata. Categoría asignada según la puntuación percentil obtenida en el Test de memoria auditiva inmediata de Cordero Pando.

- Nivel de comprensión de lectura. Categoría asignada según la puntuación percentil obtenida en el Test de complejidad lingüística progresiva de Condemarín, Allende y Milicia. 
A su vez se asumieron como variables intervinientes, el Grado de estudio (alumnos de 5to y 6to grado de educación primaria), lugar de residencia (distrito de San Juan de Lurigancho, Lima y Distrito de San Mateo, Huarochirí) y sexo.

Como instrumentos de investigación se utilizaron la Prueba de Complejidad Lingüística Progresiva (CLP) Nivel 5 y 6. Forma A. Elaborada por Condemarín, Allende y Milicic (1991), adaptada para Lima Metropolitana fue realizada Delgado A. (2005). El objetivo fue evaluar el Nivel de Comprensión de Lectura, que presenta 8 niveles de aplicación. La forma de administración puede ser individual o colectiva.

Descripción del Instrumento. La Prueba de Comprensión Lectora de Complejidad Lingüística Progresiva (CLP) Formas Paralelas, es un instrumento estandarizado para medir la capacidad de lectura principalmente en las etapas correspondientes a los años de educación general básica (1er grado de primaria a 3er año de secundaria). Fue elaborada y estandarizada en Chile por Condemarín, Allende y Milicic en 1991.

La organización de la prueba está basada en niveles de lectura, los que corresponden en grandes líneas a los grados escolares. El logro de los niveles puede ser considerablemente más lento; sin embargo, se espera que al finalizar el 3er año de secundaria se alcance el dominio de todos los niveles. La prueba está dividida en ocho niveles de lectura, cada nivel está constituido de modo que presente una dificultad creciente desde el punto de vista lingüístico, paralelamente se incrementa en la dificultad de la comprensión. Para determinar los niveles, se han tenido en cuenta tres operaciones específicas de la lectura y cuatro áreas de aplicación. Las operaciones específicas de la lectura que se han considerado en la prueba, son tres:

- Traducir los signos escritos a sus correspondientes signos orales;

- Dar a cada palabra el sentido correcto dentro del texto y retener su significación;

- Descubrir, retener y manejar las relaciones que guardan entre sí los diversos elementos del texto y determinar sentidos globales.

La prueba está referida fundamentalmente a las habilidades requeridas por la segunda y tercera operación. Los niveles de lectura elegidos para el presente estudio fue: Quinto Nivel y Sexto Nivel - Forma A. Las áreas de lectura son: de la palabra, de la oración o frase, de párrafo o texto simple y el texto complejo. Cada nivel está construido sobre la base de un conjunto de habilidades de lectura referidas a cada una de las áreas y operaciones que se señalaron anteriormente. Con respecto a los niveles de quinto y sexto la prueba examina lo siguiente:

Quinto nivel de lectura. En este nivel el conjunto de habilidades determinado para los últimos cinco niveles se aplica a tres textos narrativos y a un texto descriptivo. En los textos aparecen sujetos individuales y colectivos de carácter concreto. En el último texto cada forma introduce personajes literarios y elementos simbólicos.

El dominio del nivel se comprueba específicamente por las habilidades siguientes:

- Categorización fina de objetos, hechos y personas,

- Interpretación de elementos simbólicos, 
- Caracterización adecuada de personajes literarios,

- Especificación del sentido de palabras y expresiones de un texto.

Sexto nivel de lectura. En este nivel de lectura se comprueba el dominio del conjunto de las habilidades propias del área del texto aplicándolas a dos narraciones (una de ellas es utilizada en el nivel anterior). En este nivel se profundizan las habilidades de los niveles anteriores poniendo énfasis en:

- ordenar los hechos de acuerdo a su secuencia,

- señalar relaciones de causa y efecto entre los hechos relatados,

- dominar las relaciones de inclusión existentes entre las afirmaciones de un texto,

- captar informaciones presentadas a través de los diálogos.

Confiabilidad. Realizado con el método test-retest: la prueba de cuarto grado alcanzó un coeficiente de correlación rho de Spearman de 0,70, la prueba de quinto grado obtuvo un coeficiente de 0,75 y la de sexto grado alcanzó un valor de 0,78 , en todos los casos los coeficientes de correlación alcanzados son estadísticamente significativos $(\mathrm{p}<.05)$.

Validez. Mediante el análisis de la validez de constructo, efectuado a través del análisis factorial confirmatorio, también en este caso el modelo de un factor se contrastó con un modelo alternativo que asumía la existencia de valores independientes entre sí, los resultados presentados en la Tabla A y el Gráfico A, indicaron que se alcanzó un valor de chi cuadrado mínimo no significativo $\left(\mathrm{X}^{2}=1,185 \mathrm{p}>0,05\right)$ y una proporción $(0,593)$ entre el chi cuadrado mínimo y los grados de libertad que permiten aceptar el modelo propuesto. Los análisis correspondientes a la obtención del índice del ajuste normalizado Delta 1 (NFI Delta 1) arrojó un valor igual a 0,995, el índice comparativo de ajuste (CFI) fue de 1000 y la media de cuadrados del error de aproximación (RMSEA) fue de 0,000. Estos hallazgos corroboran el modelo propuesto de un factor como válido, con lo cual se concluye que la prueba de Comprensión Lectora de Complejidad Lingüística ProgresivaForma A para quinto grado de primaria, tiene validez de constructo.

En el caso del análisis de la validez de constructo de la prueba para el sexto grado de primaria, efectuado a través del análisis factorial confirmatorio, el modelo de un factor se contrastó con un modelo alternativo que asumía la existencia de valores independientes entre sí. Los resultados presentados en la Tabla B y el Gráfico B, indican un valor de chi cuadrado mínimo no significativo $\left(\mathrm{X}^{2}=11.942 \mathrm{p}>0,05\right)$ y un valor aceptable $(1,327)$ correspondiente a la proporción del chi cuadrado mínimo y los grados de libertad, lo cual indica que el modelo propuesto es adecuado. Los análisis adicionales del índice del ajuste normalizado Delta 1 (NFI Delta 1) ascendió a .984, el índice comparativo de ajuste (CFI) fue de 0,996 y la media de cuadrados del error de aproximación (RMSEA) fue de 0,021, hallazgos que corroboran que el modelo propuesto de un factor es válido, con lo cual se concluye que la prueba de Comprensión Lectora de Complejidad Lingüística Progresiva para sexto grado de primaria - Forma A, presenta validez de constructo.

Test de Memoria Auditiva Inmediata (MAl). Elaborado por Cordero Pando, A (1978). La adaptación para Lima Metropolitana fue realizada por Dioses, Manrique y Segura 
(2002). Tiene como objetivo evaluar la memoria lógica, numérica y asociativa a partir de estímulos auditivos. La administración puede ser Individual y colectiva. La duración es variable, según la edad y nivel de los sujetos, no se exige un tiempo fijo de ejecución en ninguna de sus partes. El tiempo para la aplicación total de la prueba se estima en cuarenta y cinco minutos, incluyendo el tiempo dedicado a instrucciones. Está dirigido a estudiantes a partir de los 8 años de edad. La prueba consta de tres partes:

Primera parte: Memoria lógica. Se presenta al sujeto dos párrafos con los cuales se intenta descubrir hasta qué punto es capaz de recordar los detalles de un relato que podría constituir el contenido de una noticia periodística de "sucesos". Los datos mantienen entre sí una coherencia significativa en cuanto están integrados en la unidad de una narración que se desarrolla lógicamente. No es tanto la reproducción literal, y en cierto modo mecánica, lo que interesa, sino el grado de fidelidad con que los datos recientemente escuchados son reproducidos.

Segunda parte: Memoria numérica. Se utilizan series de dígitos que el sujeto debe repetir, como primer ensayo, el mismo orden en que le son expuestos y en el segundo ensayo, en orden inverso.

Tercera parte: Memoria asociativa. Consta de diez pares de palabras que se presentan al sujeto en tres ocasiones distintas (cambiando cada vez el orden de presentación). Luego el sujeto debe recordar la pareja de cada palabra, a partir de la lectura de la primera palabra del par.

Confiabilidad. Su establecimiento tuvo por objetivo estimar el error existente en la medida y su indicador el coeficiente de confiabilidad. El cálculo empírico de este coeficiente se efectuó con la técnica de consistencia interna, entendida como intercorrelación entre los ítems, utilizando el Coeficiente Alfa de Cronbach, calculado en la muestra de participantes en el estudio, obteniéndose como se muestra en la tabla $\mathrm{C}$, un alfa cercano a 0.80 , el mismo que permite afirmar que la prueba proporciona puntajes confiables.

Validez. La calidad individual de los ítems fue valorada estimando el poder discriminativo de cada reactivo. Se realizó el análisis de ítem con la técnica correlación ítem-total (ítem- subtest, ya que la prueba contiene subtest e ítem-test). Todos los valores obtenidos fueron superiores a 0.20 , el mismo que es el valor mínimo indicador de la capacidad discriminativa de un ítem para su aceptación en la conformación de una prueba (Morales, 1988). La validez del instrumento fue juzgada mediante validez de contenido, mediante la cual se determinó que la muestra de reactivos era representativa del universo de ítems referentes a la memoria auditiva inmediata para niños de quinto y sexto grado de primaria. Para efectuar esta validez se recurrió a jueces o expertos: psicólogos y profesores de educación primaria, quienes dieron su opinión favorable con respecto a los reactivos e instrucciones.

Análisis estadístico de los datos. El procesamiento y análisis de los datos se realizó mediante el programa estadístico SPSS v. 14, de acuerdo con las técnicas estadísticas descriptivas e inferenciales para verificar las hipótesis planteadas, utilizando las medidas de tendencia central y de variabilidad, como: media, varianza y desviación estándar y a nivel inferencial se utilizaron: la Prueba U de Mann-Whitney, para probar si dos grupos 
independientes han sido tomados de la misma población y la prueba Rho de Spearman para establecer la correlación entre puntajes de escala ordinal de las pruebas aplicadas.

\section{RESULTADOS}

En primer lugar se verá la comprensión de lectura según el lugar de procedencia, grado de estudios y el sexo, en estudiantes que cursan el 5to y 6to grado de primaria. En segundo lugar se examina la relación que existe entre el nivel de comprensión de lectura y memoria auditiva inmediata en niños de zonas rural y urbana de Lima. El análisis de los datos fue utilizando la U de Mann-Whitney ya que según la Prueba de Kolmogorov-Smirnov el valor encontrado es mayor al 0,05 lo que indica que los datos no se distribuyen normalmente razón por la cual no podemos trabajar con estadísticos paramétricos. La U de Mann-Whitney es un estadístico cuya potencia es similar a la Prueba $t$ de student y permite comparar grupos en función de rangos. Para nuestro caso, dado que los grupos estudiados son mayores de 20, hemos utilizado la fórmula modificada por las ligas.

Tabla N. ${ }^{0}$ 1. Resultados en Memoria Auditiva Inmediata (MAI), de los alumnos de 5to y 6to grado de primaria de Huarochirí (Hua) y Lima (Lim).

\begin{tabular}{|c|c|c|c|c|c|c|c|c|}
\hline & \multicolumn{8}{|c|}{ Memoria Auditiva Inmediata } \\
\hline & \multicolumn{2}{|c|}{$\begin{array}{c}\text { Memoria } \\
\text { Lógica }\end{array}$} & \multicolumn{2}{|c|}{$\begin{array}{c}\text { Memoria } \\
\text { Numérica }\end{array}$} & \multicolumn{2}{|c|}{$\begin{array}{c}\text { Memoria } \\
\text { Asociativa }\end{array}$} & \multicolumn{2}{|c|}{$\begin{array}{c}\text { Test Total } \\
\text { MAI }\end{array}$} \\
\hline & Hua & Lim & Hua & $\mathrm{Lim}$ & Hua & Lim & Hua & Lim \\
\hline Media & 10,20 & 10,66 & 8,66 & 9,98 & 23,62 & 25,07 & 42,94 & 45,25 \\
\hline Mediana & 10,00 & 10,00 & 8,00 & 10,00 & 24,00 & 26,00 & 42,50 & 46,00 \\
\hline & 10 & 10 & 8 & 10 & 30 & 30 & 40 & 46 \\
\hline $\begin{array}{l}\text { Desviación } \\
\text { estándar }\end{array}$ & 4,095 & 4,237 & 2,318 & 2,754 & 5,369 & 4,351 & 8,942 & 7,655 \\
\hline Varianza & 16,769 & 17,951 & 5,371 & 7,585 & 28,822 & 18,930 & 79,958 & 58,596 \\
\hline
\end{tabular}

$\mathrm{N}=228$

En el Tabla N. ${ }^{\circ} 1$ con respecto a los resultados de la prueba Memoria Auditiva Inmediata (MAI), se observa que los alumnos de Lima presentan en memoria lógica una media $(10,66)$ ligeramente mayor a los de Huarochirí $(10,20)$; en el caso de memoria numérica los alumnos de Lima presentan una media $(9,98)$ mayor que la media de los estudiantes de Huarochirí $(8,66)$ y en memoria asociativa los estudiantes de Lima obtienen una media $(25,07)$ mayor que los de Huarochirí. La puntuación en memoria auditiva inmediata (test total) más frecuente fue 40 en Huarochirí y 46 en Lima. Además la mediana de las puntuaciones obtenidas por los estudiantes de Huarochirí es de 42,50 y en Lima 46,0; también se observa que los puntos obtenidos por los alumnos de Huarochirí se dispersan más de la media que los alumnos limeños. 
Tabla N. ${ }^{0}$ 2. Resultados de los promedios en comprensión lectora, de los alumnos de 5to y 6to grado de primaria de Huarochiri (Hua) y Lima (Lim).

\begin{tabular}{lrrrr}
\cline { 2 - 5 } & \multicolumn{4}{c}{ Comprensión lectora } \\
\cline { 2 - 5 } & \multicolumn{2}{c}{ 5to } & \multicolumn{2}{c}{ 6to } \\
\cline { 2 - 5 } & \multicolumn{1}{c}{ Hua } & \multicolumn{1}{c}{ Lim } & \multicolumn{1}{c}{ Hua } & \multicolumn{1}{c}{ Lim } \\
\hline Media & 13,93 & 15,34 & 13,96 & 15,05 \\
Mediana & 13,00 & 15,00 & 12,00 & 14,00 \\
Moda & 11 & 17 & 11 & 11 \\
Desviación estándar & 6,74 & 7,56 & 6,86 & 5,62 \\
Varianza & 45,44 & 57,09 & 47,04 & 31,63 \\
\hline
\end{tabular}

$\mathrm{N}=228$

En el Tabla N. ${ }^{\circ} 2$ se registran los resultados de la prueba de comprensión lectora progresiva (CLP), observándose que los alumnos de Lima presentan un promedio mayor en ambos grados; así para 5to $\bar{X}=15,34$ y para 6to $\bar{X}=15,05$. En relación con los alumnos de Huarochirí corresponde para 5to $\bar{X}=13,93$ y para 6to $\bar{X}=13,96$. Además, el puntaje que más se repitió en Huarochirí fue 11 en 5to y 6to y en Lima 17 en 5to y 11 en 6to; asimismo, la mediana de las puntuaciones obtenidas por los alumnos de Huarochirí es 13 para el 5to y 12 para el 6to mientras en Lima fue de 15 para el 5to grado y 14 para el 6to grado; finalmente se observa que los puntos obtenidos por los alumnos de Huarochirí se dispersan más de la media que los alumnos limeños.

Tabla N. ${ }^{0}$ 3. Resultados en Memoria Auditiva Inmediata (MAI) de los alumnos de 5to y 6to grado de primaria, según lugar y sexo.

\begin{tabular}{lrrrrrrrr}
\hline & \multicolumn{3}{c}{ Memoria lógica } & \multicolumn{3}{c}{ Memoria numérica } \\
& Huarochirí & \multicolumn{1}{c}{ Lima } & \multicolumn{1}{c}{ Huarochirí } & \multicolumn{2}{c}{ Lima } \\
\cline { 2 - 10 } & \multicolumn{1}{c}{$\mathrm{m}$} & $\mathrm{f}$ & $\mathrm{m}$ & $\mathrm{f}$ & $\mathrm{m}$ & $\mathrm{f}$ & $\mathrm{m}$ & $\mathrm{f}$ \\
\hline Media & 10,7 & 10,6 & 10,6 & 9,8 & 8,8 & 8,6 & 9,9 & 9,8 \\
Mediana & 10,0 & 10,0 & 10,0 & 10,0 & 8,0 & 8,0 & 10,0 & 10,0 \\
Moda & 10 & 7 & 10 & 11 & 8 & 8 & 8 & 11 \\
Desviación estándar & 3,97 & 4,3 & 4,1 & 4,4 & 2,2 & 2,4 & 2,9 & 4,4 \\
Varianza & 15,7 & 18,1 & 16,6 & 19,2 & 4,9 & 5,9 & 8,3 & 19,2 \\
\hline & \multicolumn{3}{c}{ Memoria asociativa } & & Test total MAI & \\
& Huarochirín & Lima & Huarochirí & Lima \\
\cline { 2 - 10 } & $\mathrm{m}$ & $\mathrm{f}$ & $\mathrm{m}$ & $\mathrm{f}$ & $\mathrm{m}$ & $\mathrm{f}$ & $\mathrm{m}$ & $\mathrm{f}$ \\
\hline Media & 23,9 & 23,4 & 24,3 & 25,8 & 43,3 & 42,6 & 44,9 & 45,6 \\
Mediana & 25,0 & 23,0 & 26,0 & 27,0 & 43,0 & 41,0 & 46,0 & 46,0 \\
Moda & 30 & 30 & 30 & 27 & 50 & 36 & 46 & 49 \\
Desviación estándar & 5,3 & 5,5 & 5,1 & 3,4 & 9,2 & 8,8 & 8,5 & 6,8 \\
Varianza & 28,3 & 29,7 & 25,5 & 11,8 & 83,7 & 77,6 & 72,2 & 46,1 \\
\hline
\end{tabular}

$\mathrm{N}=228$ 
En el Tabla N. ${ }^{\circ} 3$ se muestra que los puntajes promedios de la prueba general de memoria auditiva inmediata (MAI), las alumnas de Lima obtuvieron un mejor promedio $(\overline{\mathbf{X}}=45,5)$ destacándose en memoria asociativa $(\overline{\mathbf{X}}=25,8)$; por el contrario, los alumnos Lima obtuvieron una media general en la misma prueba menor que sus pares femeninos de Lima $(\overline{\mathbf{X}}=44,9)$, y destacan memoria lógica $(\overline{\mathbf{X}}=10,6)$. Además, el puntaje que más se repitió en el caso de las alumnas y alumnos de Huarochirí fue 50 y 36, y en el caso de Lima 46 y 49; asimismo la mediana de las puntuaciones obtenidas por las alumnas de Huarochirí fue de 43,0 y en el caso de los alumnos 41,0 y en Lima la mediana fue de 46 para ambos sexos. Finalmente se observa que los puntos obtenidos por los alumnos de Huarochirí se dispersan más de la media que los alumnos limeños.

Tabla N. ${ }^{\circ}$ 4. Resultados de la Memoria Auditiva Inmediata (MAI), de los alumnos según lugar y grado escolar (5to y 6to grado).

\begin{tabular}{lrrrrrrrr}
\hline & \multicolumn{3}{c}{ Memoria lógica } & \multicolumn{3}{c}{ Memoria numérica } \\
& Huarochirí & \multicolumn{2}{c}{ Lima } & \multicolumn{1}{c}{ Huarochirí } & \multicolumn{2}{c}{ Lima } \\
\hline & 5 & 6 & 5 & 6 & 5 & 6 & 5 & 6 \\
\hline Media & 10,2 & 11,1 & 9,9 & 10,6 & 8,5 & 8,8 & 9,8 & 10,2 \\
Mediana & 10,0 & 10,0 & 10,0 & 10,0 & 8,0 & 8,0 & 10,0 & 10,0 \\
Moda & 10,0 & 7,0 & 10,0 & 9,0 & 8,0 & 8,0 & 10,0 & 9,0 \\
Desviación estándar & 3,9 & 4,2 & 4,1 & 4,4 & 2,3 & 2,4 & 2,7 & 2,8 \\
Varianza & 15,2 & 18,0 & 16,8 & 19,4 & 5,2 & 5,6 & 7,2 & 8,1 \\
\hline & \multicolumn{3}{c}{ Memoria asociativa } & & Test total MAI & \\
& Huarochirín & Lima & Huarochirí & Lima \\
\hline & 5 & 6 & 5 & 6 & 5 & 6 & 5 & 6 \\
\hline Media & 23,2 & 24,0 & 24,1 & 26,2 & 41,9 & 43,9 & 43,8 & 47,0 \\
Mediana & 23,0 & 25,0 & 26,0 & 27,0 & 41,0 & 44,0 & 44,0 & 48,0 \\
Moda & 23,0 & 30,0 & 26,0 & 30,0 & 40,0 & 50,0 & 46,0 & 49,0 \\
Desviación estándar & 4,0 & 6,4 & 4,6 & 3,8 & 7,3 & 10,2 & 7,7 & 7,2 \\
Varianza & 15,9 & 40,5 & 21,0 & 14,5 & 52,7 & 103,6 & 59,8 & 52,5 \\
\hline
\end{tabular}

$\mathrm{N}=228$

En la Tabla N. ${ }^{\circ} 4$ se registran los resultados en la prueba memoria auditiva inmediata (MAI), observándose que los alumnos de 6to grado de Lima obtuvieron un alto promedio general $(\bar{X}=47,0)$, destacándose también en memoria asociativa y numérica $(\bar{X}=26,2$ y $\overline{\mathbf{X}}=10,2)$, respectivamente; por el contrario los alumnos de 5to grado de Huarochirí obtuvieron una media menor $(\bar{X}=41,9)$. Además, el puntaje que más se repitió en el caso de las 5to y 6to grado de Huarochirí fue 40 y 50, y en el caso de Lima 46 y 49; asimismo, el $50 \%$ de los alumnos de 5to y 6to grado de Huarochirí obtiene un puntaje por encima de 41 y 44 y en el caso de Lima es 44 y 48 . Finalmente se observa que los puntos obtenidos por los alumnos de 5to y 6to grado Huarochirí se dispersan más de la media que los alumnos de Lima. 
A continuación se presentan los resultados inferenciales sobre los datos obtenidos en la muestra con el propósito de comprobar las hipótesis planteadas.

Tabla N. ${ }^{0}$ 5. Resultados de la Prueba de Kolmogorov-Smirnov de Bondad de ajuste a la curva normal.

\begin{tabular}{lccccc}
\hline & $\begin{array}{c}\text { Memoria } \\
\text { lógica }\end{array}$ & $\begin{array}{c}\text { Memoria } \\
\text { numérica }\end{array}$ & $\begin{array}{c}\text { Memoria } \\
\text { asociativa }\end{array}$ & MAI & CLP \\
\hline Z de Kolmogorov-Smirnov & 1,405 & 2,077 & 1,905 & 0,896 & 1,842 \\
Significación asintótica bilateral $(p)$ & 0,038 & 0,000 & 0,001 & 0,398 & 0,002 \\
\hline
\end{tabular}

$\mathrm{N}=228$

La Tabla N. ${ }^{\circ}$ 5, nos ofrece el estadístico de K-S siendo el valor de $Z=1,405$ en el caso de Memoria Lógica, $Z=2,077$ en Memoria Numérica, $Z=1,905$ en memoria asociativa, $Z=0,896$ en memoria auditiva inmediata y $Z=1,842$ en comprensión lectora. Además se observa que solo el nivel crítico de la memoria auditiva inmediata (Significación asintótica bilateral) es mayor que 0,398, y que por el contrario en el caso de memoria lógica, numérica, asociativa y comprensión lectora son menores que 0,05 ; por lo tanto, recházanos la hipótesis de normalidad y concluimos que las puntuaciones de estas variables no se ajustan a una distribución normal.

Tabla N. ${ }^{\circ}$ 6. Coeficiente Rho de Spearman en la relación entre memoria auditiva inmediata y comprensión lectora.

\begin{tabular}{lccccc}
\hline & $\begin{array}{c}\text { Memoria } \\
\text { lógica }\end{array}$ & $\begin{array}{c}\text { Memoria } \\
\text { numérica }\end{array}$ & $\begin{array}{c}\text { Memoria } \\
\text { asociativa }\end{array}$ & $\begin{array}{c}\text { Test total } \\
\text { MAI }\end{array}$ \\
\hline $\begin{array}{l}\text { Comprensión } \\
\text { Lectora }\end{array}$ & $\begin{array}{c}\text { Coeficiente } \\
\text { de correlación }\end{array}$ & 0,342 & 0,200 & 0,290 & 0,430 \\
& $p$ & 0,000 & 0,002 & 0,000 & 0,000 \\
\hline
\end{tabular}

$p=0.05$

En la Tabla N. ${ }^{\circ} 6$ se observa que entre comprensión lectora y memoria lógica se presenta una correlación moderada (rho $=0,342$ ), entre comprensión lectora y memoria numérica se presenta una correlación débil (rho $=0,200)$, entre comprensión lectora y memoria asociativa se presenta una correlación moderada (rho $=0,290)$ y entre comprensión lectora y el test completo de memoria auditiva inmediata se presenta una correlación moderada $($ rho $=0,430)$. En todos los casos se observa un nivel de significación bilateral de 0,01 y el número de casos observado fue 228 . 
Tabla N. ${ }^{\circ}$ 7. Resultados de la $U$ de Mann-Whitney que establece las diferencias entre los alumnos de Lima y Huarochirí en memoria auditiva inmediata y comprensión lectora.

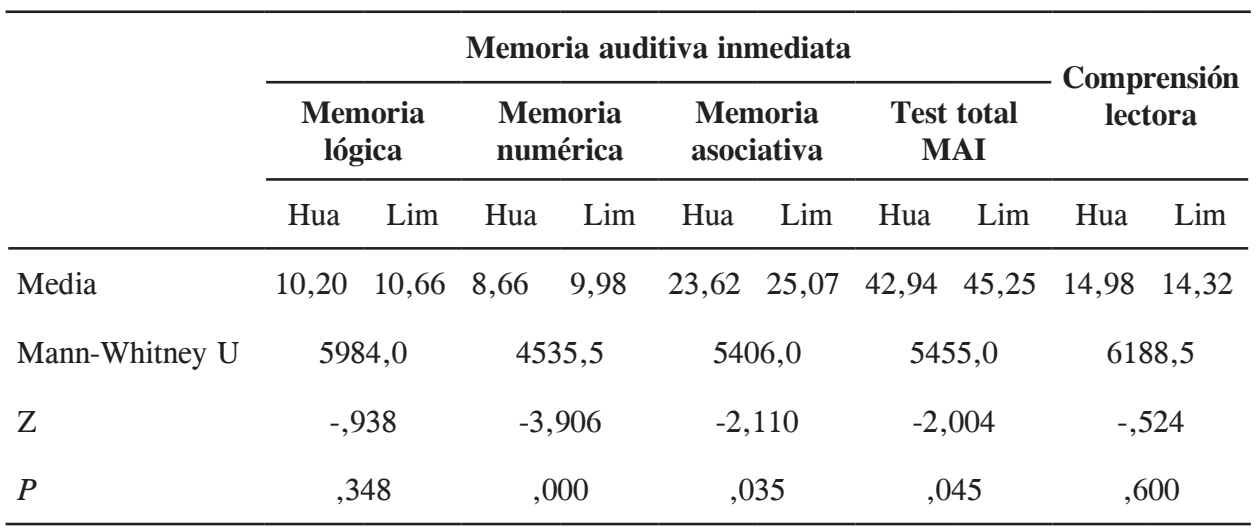

Variable agrupamiento: lugar

La Tabla N. ${ }^{o} 7$ muestra resultados del estadístico $U$ de Mann-Whitney estableciendo las deferencias entre las medias de memoria auditiva inmediata y comprensión lectora. El nivel crítico bilateral $(p)$ es inferior a 0,05 en el caso de memoria numérica, memoria asociativa y en memoria auditiva inmediata (test total); por lo tanto existen diferencias significativas entre los alumnos de Lima y Huarochirí en esas áreas. En los casos de Memoria Lógica y Comprensión Lectora no existen diferencias significativas entre los alumnos de Lima y Huarochirí.

Tabla N. ${ }^{\circ}$ 8. Resultados de la $U$ de Mann-Whitney para establecer las diferencias entre los alumnos y alumnas en memoria auditiva inmediata y comprensión lectora.

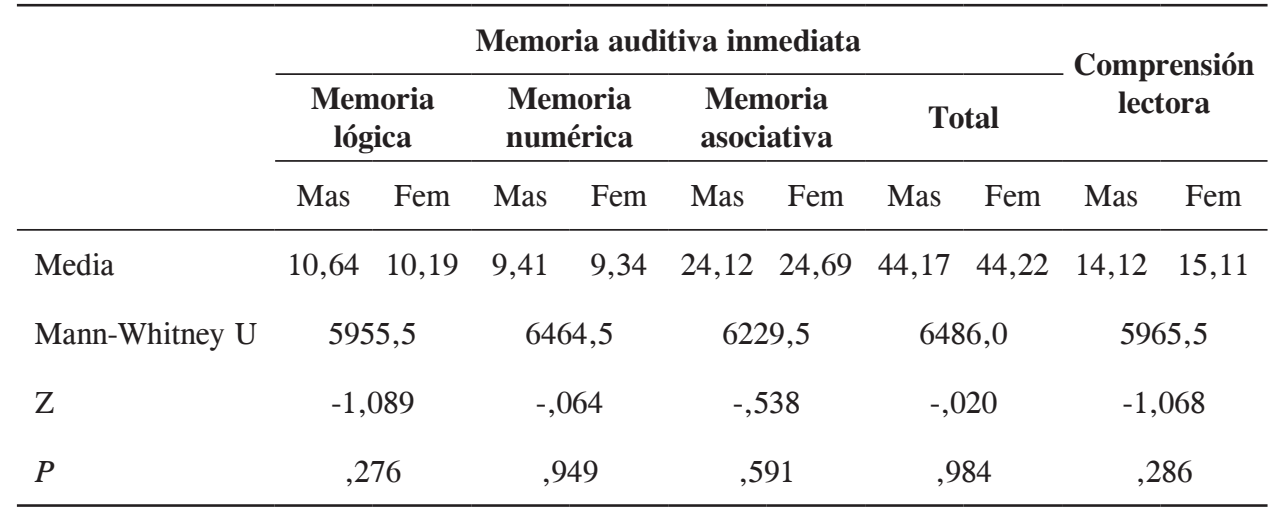

Variable agrupamiento: sexo 
La Tabla N. ${ }^{\circ} 8$ muestra resultados de la $U$ de Mann-Whitney que establece deferencias entre las medias de Memoria Auditiva Inmediata y Comprensión Lectora entre las alumnas y alumnos. Se observa un nivel crítico bilateral $(p)$ superior a 0,05 en todas las áreas observadas, lo cual indica que no existen diferencias significativas entre los alumnos y alumnas en las áreas que conforman la Memoria Auditiva Inmediata y en Comprensión Lectora.

Tabla N. ${ }^{\circ}$ 9. Resultados de la $U$ de Mann-Whitney para establecer las diferencias entre la memoria auditiva inmediata y comprensión lectora según grado escolar.

\begin{tabular}{|c|c|c|c|c|c|c|c|c|c|c|}
\hline & \multicolumn{8}{|c|}{ Memoria auditiva inmediata } & \multirow{2}{*}{\multicolumn{2}{|c|}{$\begin{array}{l}\text { Comprensión } \\
\text { lectora }\end{array}$}} \\
\hline & \multicolumn{2}{|c|}{$\begin{array}{c}\text { Memoria } \\
\text { lógica }\end{array}$} & \multicolumn{2}{|c|}{$\begin{array}{l}\text { Memoria } \\
\text { numérica }\end{array}$} & \multicolumn{2}{|c|}{$\begin{array}{c}\text { Memoria } \\
\text { asociativa }\end{array}$} & \multicolumn{2}{|c|}{$\begin{array}{c}\text { Test total } \\
\text { MAI }\end{array}$} & & \\
\hline & $5^{\circ}$ & $6^{0}$ & $5^{\circ}$ & $6^{0}$ & $5^{\circ}$ & $6^{0}$ & $5^{\circ}$ & $6^{0}$ & $5^{\circ}$ & $6^{0}$ \\
\hline Media & 10,00 & 10,84 & 9,24 & 9,52 & 23,72 & 25,13 & 42,96 & 45,48 & 9,54 & 19,88 \\
\hline Mann-Whitney U & \multicolumn{2}{|c|}{6055,0} & \multicolumn{2}{|c|}{6078,0} & \multicolumn{2}{|c|}{4988,0} & \multicolumn{2}{|c|}{5130,5} & \multicolumn{2}{|c|}{603,0} \\
\hline $\mathrm{Z}$ & \multicolumn{2}{|c|}{,- 889} & \multicolumn{2}{|c|}{,- 851} & \multicolumn{2}{|c|}{$-3,043$} & \multicolumn{2}{|c|}{$-2,745$} & \multicolumn{2}{|c|}{$-11,859$} \\
\hline $\mathrm{P}$ & \multicolumn{2}{|c|}{,374 } & \multicolumn{2}{|c|}{,395 } & \multicolumn{2}{|c|}{, 002} & \multicolumn{2}{|c|}{, 006} & \multicolumn{2}{|c|}{, 000} \\
\hline
\end{tabular}

Variable agrupamiento: grado de estudio.

La Tabla N. ${ }^{\circ} 9$ presenta los resultados del estadístico $U$ de Mann-Whitney que establece diferencias entre las medias de memoria auditiva inmediata y comprensión lectora entre los alumnos de 5to y 6to grado de primaria. Se observó un nivel crítico bilateral $(p)$ menor a 0,05 en memoria asociativa, memoria auditiva inmediata (test completo) y comprensión lectora; esto indica que existen diferencias significativas entre los alumnos de 5to y 6to de primaria en estas áreas. En los casos de memoria lógica y memoria numérica no existen diferencias significativas entre los alumnos de $5^{\circ}$ y $6^{\circ}$ de primaria en las áreas mencionadas.

\section{DISCUSIÓN}

En primer lugar, al analizar los resultados en memoria auditiva inmediata teniendo en cuenta la variable lugar donde viven (Lima o Huarochirí) observamos resultados ligeramente mejores en memoria lógica y mayores diferencias en memoria numérica, asociativa y memoria auditiva inmediata a favor de los alumnos de Lima (Tabla N. ${ }^{\circ} 1$ ). Estos resultados evidencian que los alumnos de Lima presentan mejores resultados para la evocación escrita de la idea esencial de una narración escuchada previamente, ya sea utilizando las mismas palabras y estructuras gramaticales, o haciendo uso de sinónimos y estructuras gramaticales equivalentes, recordar series numéricas escuchadas previamente escribiéndolas en forma directa o inversa, así como la evocación escrita de palabras que estaban asociadas a otras. 
Lo anterior coincide con los resultados de García y Martín (1987), pues en el caso de la memoria a corto plazo, se activa el mecanismo de asociación, secuenciación, linealidad y recuerdo del texto, siguiendo la trayectoria o disposición lógica de la lectura estructurada a medida que se va leyendo.

Al observar los promedios obtenidos en comprensión lectora por parte de los estudiantes de 5to y 6to grado de primaria teniendo en cuenta la variable lugar donde viven (Huarochirí o Lima), se puede apreciar la existencia de diferencias en ambos grupos (Tabla N. ${ }^{\circ} 2$ ), resultado similar a los hallados por Carreño (2000), que encontró diferencias en los rendimientos del aprendizaje de la lectura según la pertenencia a departamentos de la costa o sierra. Estos resultados coinciden con los hallados en la Evaluación Nacional 2004 (Ministerio de Educación, 2005), en el cual los alumnos de sexto grado de educación primaria de instituciones educativas estatales del ámbito urbano lograron mayores niveles de rendimiento en comprensión de textos que sus pares de ámbitos rurales.

Según los datos obtenidos en memoria auditiva inmediata según lugar y sexo (Tabla N. ${ }^{\circ} 3$ ) se observa que las alumnas de Lima tienen mejor promedio en memoria auditiva inmediata (Test completo) y en el subtest de memoria asociativa. En el caso de los hombres sólo se presentan diferencias a favor de ellos en los resultados de memoria lógica y numérica. Sobre esto, Peñacoba (2006), plantea que las niñas suelen mostrar un perfil madurativo ligeramente más avanzado que los niños en el área cognitivo-lingüística.

Al comparar los resultados según Memoria Auditiva Inmediata y grado de estudio (Tabla $\mathrm{N}^{0} 4$ ), se observa que los alumnos de 6to grado (de Lima y Huarochirí) obtienen mejores promedios en todos los subtest, hay diferencia en las puntuaciones de alumnos de sexto grado con respecto a los de quinto grado. Si tenemos en cuenta que el promedio de edad de los alumnos de quinto grado es 9,96 y el de los alumnos de sexto grado 11,04; podemos relacionar estos resultados con lo que señala García y otros (1995), que la velocidad con que se realizan diversos procesos cognitivos (como la articulación y codificación de palabras) crece a medida que la edad aumenta, especialmente entre los 8 y 12 años de edad, repercutiendo favorablemente en otros procesos de orden superior, como la retención o la comprensión, lo cual incide en la memoria que presenta un crecimiento gradual en el desarrollo desde los 6 a los 19 años de edad.

Al realizar las comparaciones de los niveles de memoria auditiva inmediata y sus componentes (Gráficos N. ${ }^{\circ}$ 1, 2, 3 y 4), se observó un mayor porcentaje de estudiantes de Huarochirí que se ubican en los niveles Inferior y Superior en el Test total de MAI y Comprensión Lectora, en comparación con estudiantes de Lima; esto se explicaría por una mayor dispersión de los datos en el caso de los alumnos de Huarochirí, que revela una mayor variabilidad en el desarrollo de competencias lectoras. Al comparar estudiantes varones y mujeres se observa que los varones presentan porcentaje mayor en el nivel Inferior en Test total de MAI, y en el caso las mujeres logran porcentaje mayor en el nivel inferior y superior en el caso de comprensión lectora. Este resultado es distinto a los hallados por Mares (1995), que constata la no existencia de dichas diferencias en niños y niñas, lo cual lleva a plantear que en el caso de la alumnas otros factores estarían participando a su favor, tales como mejores hábitos de estudio, mayor motivación o apoyo de los padres. 
También se constató una correlación moderada entre la comprensión lectora y la memoria auditiva inmediata (ver Tabla N. ${ }^{\circ}$ 6). Luego al analizar la relación entre los componentes de la memoria auditiva inmediata y la comprensión lectora, se observó que la memoria lógica y la memoria asociativa presentan una correlación moderada con la comprensión lectora. En el caso de la memoria numérica la correlación es débil con la comprensión lectora. Esto significa que existe una relación directa moderada entre la capacidad para la evocación de la idea esencial de una narración o de pares de palabras y la comprensión lectora, en el grupo de estudiantes de Lima y Huarochirí. Además, se observó una relación directa débil entre la capacidad para la evocación de series numéricas y la comprensión lectora, en estudiantes de Lima y Huarochirí.

Los datos obtenidos nos permiten señalar que de acuerdo con el lugar donde viven los sujetos, no existen diferencias significativas en los resultados de memoria lógica, memoria asociativa, memoria auditiva inmediata (test completo) y comprensión lectora (Tabla N. ${ }^{\circ}$ 7), salvo en memoria numérica. En el caso de analizar las diferencias según el sexo (Tabla N. ${ }^{\circ}$ ), se obtiene que no existen diferencias significativas en los resultados en memoria auditiva inmediata y comprensión lectora de los alumnos y alumnas. Finalmente en el caso de apreciar las diferencias en función del grado de estudio se observa (Tabla N. ${ }^{\circ}$ 9) que existen diferencias significativas entre los promedios en memoria asociativa, memoria auditiva y comprensión lectora.

Los resultados obtenidos pueden interpretarse en que la memoria auditiva inmediata permite ir asociando y evocando (posteriormente), personajes, objetos y lugares con los contenidos, acciones o escenas que se suceden en el texto. Este proceso de asociación y evocación permite la construcción de interconexiones significativas, entre las distintas partes integrantes de un texto leído con el consiguiente beneficio de comprensión lectora. Cabe señalar que en el grupo de sujetos estudiados, la memoria auditiva inmediata y sus componentes memoria asociativa y memoria lógica, tienen un rol importante en el proceso de comprensión lectora.

\section{CONCLUSIONES}

De acuerdo con el análisis de los resultados, podemos concluir lo siguiente:

En relación con la hipótesis general del presente estudio se constató que existe una correlación moderada $(\mathrm{Rho}=0,43)$ entre los puntajes del test comprensión lectora y el test de memoria auditiva inmediata (MAI)

1. Se rechaza la hipótesis $\mathrm{H} 1$ al no encontrar diferencias significativas en el nivel de comprensión de lectura entre los estudiantes de 5to y 6to grado de primaria de Lima y Huarochirí.

2. Se acepta la hipótesis $\mathrm{H} 2$ al encontrar diferencias significativas en el nivel de memoria auditiva inmediata entre los alumnos 5to y 6to grado de primaria de Huarochirí y Lima.

3. Se rechaza la hipótesis $\mathrm{H} 3$ al no encontrar diferencias significativas en comprensión lectora en alumnos de 5to y 6to grado de primaria de zonas Huarochirí y Lima, según el género. 
4. Se rechaza la hipótesis $\mathrm{H} 4$, pues no existen diferencias significativas en memoria auditiva inmediata, en alumnos de 5to y 6to grado de primaria de Huarochirí y Lima, según el género.

5. Se acepta la hipótesis H5, pues existen diferencias significativas en comprensión lectora entre los alumnos de 5to grado y 6to grado de primaria de Huarochirí y Lima.

6. Se acepta la hipótesis H6, pues existen diferencias significativas en memoria auditiva inmediata entre alumnos de 5to y 6to grado de primaria de zonas Huarochirí y Lima.

Basados en los resultados, podemos decir que en el logro de la comprensión lectora juega un papel importante la memoria auditiva inmediata y de manera específica la memoria lógica tendría más importancia en la comprensión de un texto. Por el contrario, no son tan relevantes factores externos como el lugar donde viven los sujetos.

A nivel teórico, los hallazgos obtenidos permiten relacionar la memoria auditiva inmediata propuesta por Cordero con el modelo desarrollado por Baddeley y Hitch sobre el subsistema Fonoarticulatorio de la Memoria de Trabajo, en ambos casos se implican la capacidad para retener información sobre los personajes, objetos, lugares, contenidos, acciones o escenas que se suceden en el texto. Esta información obtenida a partir de la decodificación de la información visual (texto) en información auditiva (lectura); lo cual hace necesario un proceso de registro fonológico que puede reactivarse mediante por un proceso de relectura de la huella, que se logra mediante el articulación de las palabras que permitan ir asociando o identificando partes del texto. Durante este proceso la memoria operativa permite realizar otras tareas relevantes en la comprensión de textos, que la memoria auditiva inmediata no plantea, estas tareas son: diferenciar la similitud fonológica o acústica de las palabras, el efecto del habla irrelevante que sucede cuando el recuerdo inmediato se ve favorecido por la presentación visual de conceptos o situaciones, el efecto de la longitud de las palabras que permite recordar la duración hablada o número de sílabas de las palabras leídas. Por tanto, podríamos concluir en futuras investigaciones el modelo propuesto por Baddeley y Hitch, sería el más adecuado para el estudio de la comprensión lectora.

\section{REFERENCIAS BIBLIOGRÁFICAS}

1. Alsina, Á. (2001). La intervención de la memoria de trabajo en el aprendizaje del cálculo aritmético. Tesis Doctoral, Universitat Autónoma de Barcelona.

2. Alliende, F. y Condemarín, M. (1982). La lectura: teoría, evaluación y desarrollo. Santiago de Chile, Andrés Bello.

3. Alliende, F., Condemarín, M. y Chadwick (1998). Comprensión de la lectura. Santiago de Chile: Andrés Bello.

4. Alliende, F., Condemarín, M. y Milicic, N. (1991). Prueba CLP. Formas paralelas. Madrid: CEPE.

5. Ausubel, D. P.; Novack, J. D. y Hanesian, H. (1983). Psicología educativa. México D.F.: Trillas. 
6. Baddeley, A. D. (1998). Memoria Humana. Teoría y Práctica. Madrid: McGrawHill.

7. Baddeley, A. D. (2003). Working memory: looking back and looking forward. En: Neuroscience, Vol. 4; 829-839.

8. Beltrán, J. (1993). Procesos, estrategias y técnicas de aprendizaje. Madrid: Editorial Síntesis.

9. Bravo, L. (1991). Psicología de las dificultades del aprendizaje escolar. Santiago de Chile: Editorial Universitaria.

10. Cabrera, F. y otros (1994). El proceso lector y su evaluación. Madrid: Editorial Laertes.

11. Callejas, J. (2008). Tema 5. Estudios correlacionales. (Material de lectura de la asignatura de Introducción a la psicología, disponible en www4.ujaen.es/ jecalle/ Descargas/tema5)

12. Carreño, C. (2000). Comprensión de lectura al finalizar primaria en niños peruanos. Tesis de Maestría, Pontificia Universidad Católica del Perú.

13. Carranza M., Celaya, G., Herrera, J. y Carezzano F. (2004). Una forma de procesar la información en los textos científicos y su influencia en la compresión. Revista Electrónica de Investigación Educativa, 6 (1). Consultado el 14 de abril de 2008 en: http://redie.uabc.mx/vol6no1/contenido-carranza.html

14. Castellano, S. (1994). Programa experimental de reeducación de la lectura comprensiva en alumnos de $2 d o$ grado de primaria del C.E. estatal N. ${ }^{\circ} 6099$. Tesis de licenciatura, UNMSM.

15. Clemente, M. y Domínguez, A. (1999). La enseñanza de la lectura. Madrid: Piramide.

16. Condemarín, M. y Chadwick, M. (1988). La escritura creativa y formal. Santiago de Chile, Andrés Bello.

17. Cordero Pando, A. (1978) Test de memoria auditiva inmediata (M.A.I.) Manual. Madrid: TEA Ediciones.

18. Crespo, A. (2002). Cognición humana. Madrid: Editorial Universitaria Ramón Areces.

19. Cueto, S., Jacoby, E., y Pollit, E. (1997). Rendimiento de niños y niñas de zonas rurales y urbanas del Perú. En Revista de Psicología de la Pontificia Universidad Católica del Perú. 14 (1), 115-133.

20. Delgado; A. (2004). "Comparación de la comprensión lectora en alumnos de $1^{\text {o }}$ a 3er. grado de primaria de centros educativos estatales y no estatales de Lima metropolitana”. Revista de Investigación en Psicología, 7(2), 87-116.

21. Delgado; A. (2005) "Comparación de la comprensión lectora en alumnos de $4^{\circ}$ a $6^{\circ}$ grado de primaria de centros educativos estatales y no estatales de Lima Metropolitana”. Revista de Investigación en Psicología de la UNMSM, 8(1), 51-85. 
22. Dioses, A., Manrique, S. y Segura K. (2002). Adaptación del test de memoria auditiva inmediata - MAI. Lima: Centro de Investigación y Publicaciones - CPAL.

23. Dioses, A. (2004). Memoria auditiva inmediata y dificultades ortográficas en niños de $5^{\circ}$ y $6^{\circ}$ grado de educación primaria de colegios de Lima. Lima: Centro de Investigación y Publicaciones - CPAL.

24. Etchepareborda, M.C. y Abad-Mas, L. (2005). Memoria de trabajo en los procesos básicos del aprendizaje. Revista de Neurología, 40 (supl.1).

25. Consultado el 22 de junio de 2008 en: http://campusvirtual.uma.es/psicoev/Profesores/ Romero/Documentos/MT\%20y\%20aprendizaje.pdf

26. Ferreiro, E. y Teberosky, A. (1988). Los sistemas de escritura en el desarrollo del niño. México D.F.: Siglo XXI.

27. Ferreiro, E. (2001). Pasado y presente de los verbos leer y escribir. México D.F.: Fondo de Cultura Económica.

28. García, J. y Martín, J. (1987). Aprendizaje comprensión y retención de textos. Madrid: UNE.

29. García, J. y otros (1995). Comprensión y adquisición de conocimientos a partir de textos. Madrid: Siglo XXI.

30. García, J. (2006) Lectura y conocimiento. Barcelona: Paidós.

31. Gutiérrez, F.; García Madruga J.A.; Elosúa, R.; Luque, J.L. y Garate, M (2002). Memoria operativa y comprensión lectora: algunas cuestiones básicas. Acción Psicológica 1, 45-68.

32. Hernández, R. (1991). Metodología de la investigación. México D.F.: Editorial Ultra.

33. Mares, F. (1995). "Efectos de un programa de estimulación sobre las habilidades básicas para el aprendizaje de la lectoescritura, en una muestra urbano marginal de Iquitos”. Tesis de licenciatura, UNMSM.

34. Matalinares, M. (2007). Lenguaje comprensivo y memoria auditiva inmediata en estudiantes de $5^{\circ}$ y $6^{\circ}$ grado de primaria de zona rural y urbana de Lima. Revista de Investigación en Psicología, 8(1), 51-85.

35. Ministerio de Educación (2001). Boletín UMC N. ${ }^{\circ}$ 9. El Perú en el primer estudio internacional comparativo de la UNESCO sobre lenguaje, matemática y factores asociados en tercer y cuarto grado. Lima: UMC - MINEDU.

36. Ministerio de Educación (2002). Boletín UMC N. ${ }^{o}$ 21. El programa internacional para la evaluación de estudiantes de la OCDE (PISA) y la participación del Perú. Lima: UMC - MINEDU.

37. Ministerio de Educación (2004). Documento de trabajo UMC 11. La evaluación de la alfabetización lectora de PISA y el rendimiento de los estudiantes peruanos. Lima: UMC - MINEDU. 
38. Ministerio de Educación (2005). Evaluación nacional de rendimiento estudiantil 2004 - Resultados. Lima: UMC - MINEDU.

39. Ministerio de Educación (2008). Evaluación censal de estudiantes 2007. Lima: UMC - MINEDU.

40. Neisser, U. (1979). Psicología cognoscitiva. México D.F.: Trillas.

41. Noriega Reynoso, R. (1998). Estudio cualitativo de los niveles de comprensión lectora de un grupo de niños deficientes y buenos lectores antes y después de un programa de intervención. Tesis de Maestría, UNMSM, Lima.

42. Peñacoba, C. y otros (2006). Teoría y práctica de psicología del desarrollo. Madrid, Editorial Universitaria Ramón Areces.

43. Pinzas, J. (1997). Metacognición y lectura. Lima: Pontificia Universidad Católica.

44. Pinzas, J. (1999). Leer mejor para enseñar mejor. Lima: Ediciones Tarea.

45. Ruiz Vargas, J. M. (1994). La memoria humana. Función y estructura. Madrid: Alianza Editorial.

46. Sánchez, H. (1988). Metodología y diseños en la investigación científica. Lima: Editorial Mantaro.

47. Siegel, S. (1986) Estadística no paramétrica. México D.F.: Trillas.

48. Tapia Mendieta, V. (1999) Efectos de un programa de estrategias cognitivas y metacognitivas, para mejorar la comprensión lectora. Revista de Investigación en Psicología, Vol. 3(4), UNMSM.

49. Vandendorpe, Ch. (2002). Del papiro al hipertexto. Ensayo sobre las mutaciones del texto y la lectura. Buenos Aires: FCE.

50. Vega, M. de (1984). Introducción a la psicología cognitiva. Madrid: Alianza.

51. Vidal, J. y Manjón, D. (2000). Dificultades de aprendizaje e intervención psicopedagógica. Madrid: EOS.

52. Yanac, E. (2000). Participación de los padres en relación a la comprensión lectora y su efecto en el mejoramiento lector en escolares de 3er grado de primaria, de nivel socioeconómico medio-bajo. Tesis de Maestría. UNMSM.

53. Yarleque, L. (1997). Desarrollo de la memoria lógica en estudiantes de cuarto a sexto grado de educación primaria de Huancayo. Perú: UNCP.

54. Yarleque, L. (1999). El desarrollo de la memoria lógica en escolares bilingües y monolingües. Tesis de magíster, UNMSM.

55. Zarzosa, S. (2003). El programa de lectura nivel 1 sobre la comprensión de lectura en niños que cursan el 3er grado de primaria de nivel socioeconómico medio y bajo. Tesis de Licenciatura, UNMSM. 